\title{
Report on the nature, characteristics, and outcomes of the Japanese healthcare system
}

\author{
Tatsuya Kondo*; MEJ Four Dimensional Health Innovation Group \\ Medical Excellence JAPAN, Tokyo, Japan.
}

\begin{abstract}
In Japan, healthcare takes a "patient-centeredness" approach to prioritize providing rational medicine for patients under the initiative of medical doctors. This approach to healthcare is based on the concept that patients should receive the correct diagnosis and optimal treatment. The present report aims to provide an overview of the specific characteristics of healthcare in Japan to healthcare management professionals in other countries. We introduce the systems within Japan's healthcare framework, particularly "medical team approach", "nutrition management", and "infection controls", as well as treatment results in Japan using objective data to inform medical doctors in management positions in other parts of the world. Collectively, these three healthcare systems comprise the "patient-centeredness" philosophy through which Japanese healthcare professionals perceive ideal patient care and act accordingly. These healthcare systems are unique to Japan and were developed in accordance with the specific framework of Japanese history, systems, and culture. This report presents the effects of "patientcenteredness" healthcare based on treatment results and performance data by making a quantitative and qualitative comparison with healthcare in Europe and the USA. Further objective evaluation revealed that Japan demonstrates positive treatment results that are comparable to those of Europe and the USA due to its "patient-centeredness" rational medical system and the availability of the "correct diagnosis and optimal treatment". These findings introduce Japan's "patient-centeredness" medical and healthcare system with a view of informing and guiding improvements in the healthcare quality of other countries and promoting future international collaborations.
\end{abstract}

Keywords: patient-centeredness, healthcare system, Japan

\section{Introduction}

The health insurance system in Japan provides universal health coverage that is funded by both taxes and individual contributions. All residents, including expatriates, are obliged to join either the employmentbased or residence-based health insurance (1). Additional private health insurance is common to complement the universal health insurance and prepare for unforeseen expenses.

Insured residents pay 30\% (children, elderly, and low-income citizens have lower coinsurance rates) of their medical and pharmaceutical costs and the insurer covers the rest, up to monthly and annual maximum limits (1). The government defines the benefits and sets the fee schedule, which is renewed every 2 years. The universal health insurance fundamentally works on a piecework system and is not compatible with treatments outside of the system. We believe that the Japanese healthcare system aims at providing rational medicine based on "patient-centeredness" to best provide correct diagnoses and optimal treatments. The system uses meticulous on-site improvements to provide patients with adequate services.

This report introduces three aspects within the system as specific examples, namely "medical team approach", "nutrition management", and "infection control". Outcomes of distinctive diseases are presented and compared with those of some European countries and the USA. This study aims to convey the noteworthy characteristics of the Japanese healthcare system to healthcare management professionals worldwide.

\section{Materials and Methods}

A survey of 131 medical doctors and healthcare professionals across 48 Japan International Hospitals, medical institutions accredited by the Medical Excellence Japan for their readiness to take in foreign patients in Japan, was conducted online between October and December 2019 to collect data on the strengths and advantages of the Japanese healthcare system. The information was narrowed down to 10 aspects: team approach, speed, quality and safety, medical education, nutrition support, infrastructure, nationwide equal accessibility, preventative and 
advanced medicine, leadership of physicians, and home doctors.

Six medical doctors with international backgrounds were interviewed to determine the strengths that could be shared internationally to promote better patient care and support. Three characteristics, "medical team approach", "nutrition management", and "infection control" were selected as noteworthy characteristics, and four healthcare professionals were interviewed regarding these specific aspects.

\section{Results and Discussion}

\section{Healthcare in Japan}

In Japan, healthcare focuses on providing treatment and care based on "patient-centeredness", prioritizing the provision of rational medicine to patients as a team under the initiative of medical doctors. In this report, "patient-centeredness" is defined as the attitude of healthcare professionals to provide ideal and beneficial care depending on the decision of the patient throughout their treatment based on the principle that patients should always receive the "correct diagnosis and optimal treatments".

Japan has nationwide training facilities for healthcare professionals and the network of university hospitals, small and large specialized hospitals, and clinics in urban and rural areas are well organized. Many large-scale hospitals are equipped with the facilities, systems, and skills that healthcare providers of a comparable standard would deliver in other medically advanced regions, such as Europe and North America. The universal healthcare reimbursement system evaluates the services provided by each facility so that services are maintained at a high level across the country. Universal health insurance ensures that these factors enable the equal availability of healthcare services nationwide. Furthermore, the low mortality rate of ischemic cerebral infarction (2) and high five-year survival rate of all carcinomas (3) show small regional differences within Japan.

The following sections will introduce the three characteristics, namely "medical team approach", "nutrition management", and "infection controls", of the healthcare system in Japan that support the foundation of healthcare and contribute indirectly to treatment results.

Unique features of Japan's medical and healthcare system

\section{Medical team approach}

The medical team approach is defined in Japan as "sharing goals and information of the patient, cooperating with and complementing each other while serving each of their roles, and providing flexible care depending on patients' situations via a wide variety of healthcare staff engaged in medical and healthcare on the premise of a high level of their expertise" (4).

Cooperation among disciplines is essential for delivering adequate treatment and care due to the increased specialization of each field and the subdivision of occupations. The Japanese national government certifies a diverse range of healthcare professionals and their professionalism is nurtured via continual training provided under the initiative of the academic societies in addition to hands-on training within the hospitals (Table 1).

An example that highlights this approach is the "clinical pathway", which has evolved independently in Japan and penetrated deep into the healthcare system. The idea of the clinical pathway originated in the USA as the "critical pathway", which began as a manufacturing process control technique. It was later introduced to the field of healthcare to optimize resources in hospitals by forecasting and managing the human resources necessary in each patient's treatment phase (5). Healthcare providers in Japan developed this process control technique into a tool to provide the best care for patients based on the concept of "patient-centeredness" using optimal resources. In Japan, the clinical pathway involves creating customized treatment plans for each patient and setting goals in each treatment phase (6). The members of the medical care team clearly understand their individual roles as well as the overall targets and goals so the development and implementation of the ideal care programs are unified. In other words, the clinical pathway is a tool for practicing a "medical team approach" that is customized to individual patients by facilitating information shared among the team.

These clinical pathways are constantly updated and improved. Hospitals establish clinical pathway committees and perform operational management activities associated with these such as developing, promoting, and reviewing the procedures (6). Moreover, academic societies and communities, such as the Japanese Society for Clinical Pathway, continually facilitate improvement in clinical pathways at associated hospitals by conducting exchanges of opinions on a

Table 1. Healthcare professionals certified with national qualifications in Japan

\begin{tabular}{lll}
\hline Doctors & Physiotherapists & Health laboratory technicians \\
Dentists & Occupational therapists & Clinical engineers \\
Pharmacists & Orthoptists & Dental hygienists \\
Health nurses & Speech therapists & Dental technicians \\
Midwives & Prosthetists & Emergency medical technicians \\
Nurses & Radiological technologists & Registered dieticians \\
Clinical laboratory technicians & & \\
\hline
\end{tabular}


nationwide basis ( 6 ).

The Japan Council for Quality Health Care sets interdisciplinary cooperation as one of the rating indices for hospitals with independent evaluations for inhospital cooperation based on "patient-centeredness". This serves as an external driving force to promote patient-centered cooperation.

In the USA, specialization in healthcare professionals' expertise is promoted from the perspective of management rationalization by healthcare organizations (7). There is a history of new occupations complementing traditional professionals and the healthcare delivery system has been built based on the combination of professionals with definite roles, centering on doctors.

In France, some medical procedures that are traditionally conducted by medical doctors have been authorized to be carried out by nurses to promote specialization in doctors' expertise due to increasingly sophisticated treatments and to control healthcare costs. The roles of nurses with and without expertise is stipulated by law ( 8 ) and a healthcare delivery system has been established in which multiple disciplines cooperate based on individual roles.

The "medical team approach", in Japan involves the division of labor, provides efficient healthcare services, has a comprehensive approach that includes patients in the team, and provides tailor-made goals for each patient. Furthermore, responsibilities of healthcare professionals overlap so that there are more opportunities for contingencies.

\section{Nutrition management}

"Nutrition management" refers to developing and implementing nutrition management plans for individual patients before and after treatment based on information obtained from the patients' physical condition and laboratory data to improve their prognosis. Appropriate nutrition management before and after treatment has been confirmed to stabilize patients' conditions and reduce mortality (9).

Many Japanese hospitals have set up systems to perform the same level of nutritional care as in Europe and the USA to ensure suitable nutrition management before and after treatment. Hospitals aim to provide treatment as well as customized nutrition care for each patient, with a focus on improving quality of life (QOL) after their discharge from hospital, based on the concept of "patient-centeredness".

A feature of "nutrition management" in Japan is that registered dieticians actively intervene in patients' nutrition management to improve their prognosis. This means individual patients receive the optimal nutrition management that considers nutrients, allergies, food forms that are easy to eat, and patients' taste and flavor preferences that are appropriate for the clinical conditions.

Registered dieticians coordinate with medical doctors to tailor the nutrition management for each individual patient. Registered dieticians check the patients' nutritional intake and use that information to make suggestions to the doctors about the content and forms of the meals and intravenous nutrition administration plans. They also talk with patients to relieve anxieties about meals after treatment and provide dietary instructions after discharge from hospital. They aim to improve the patients' prognosis and QOL following discharge (10).

One example of customized care to patients is thickening liquids. Post-stroke patients have a greater risk of developing pneumonia due to aspirating liquid. Registered dieticians thicken water to make it easier to drink. Since the required thickness differs according to each patient, the thickness of the water is carefully and gradually reduced as each patient recovers (11).

In Japan, registered dieticians are keenly aware of deferring to and supporting patients' wishes when they want to consume food orally. They emphasize the aspect of a hospital diet as a clinical diet to boost the nutritional intake as well as making meals enjoyable. For example, meals are provided at temperatures at which patients can enjoy them by using serving wagons with heat insulation and a cooling function (11). Moreover, some hospitals promote enjoyable meals using decorative ingredients and colors and incorporating seasonal ingredients to stimulate the appetites of patients who are reluctant to eat (11). Studies have shown that food intake increases and the effects of treatment improve when patients are willing to consume meals (12).

In Japan, "nutrition management" is supported by on-site activities, such as nutrition screening, development and review of nutrition management plans, and establishment of nutrition support teams, that are conducted at each hospital. There is a reimbursement system for "nutrition management" to promote these efforts. The same nutrition management system has been adopted by hospitals across the country since this system has come to serve as a guideline for hospitals to promote "nutrition management".

Many registered dieticians support "nutrition management" in Japan. In December 2019, the total number of registered dieticians in Japan was 244,487. The number of registered dieticians in Japan is $193 / 100,000$ people (13) and is significantly higher than that in the USA $(32 / 100,000$ people), which is considered an advanced country in terms of nutrition management (14) (Figure 1).

Due to their abundance, registered dieticians are able to provide highly individual nutrition care for each patient. This could deliver benefits such as improved prognosis for recovery and greater patient satisfaction. An example of improved prognosis is the case of outcomes for intensive care unit (ICU) patients who require fast and meticulous nutrition management. Organizing a nutrition management system that includes 


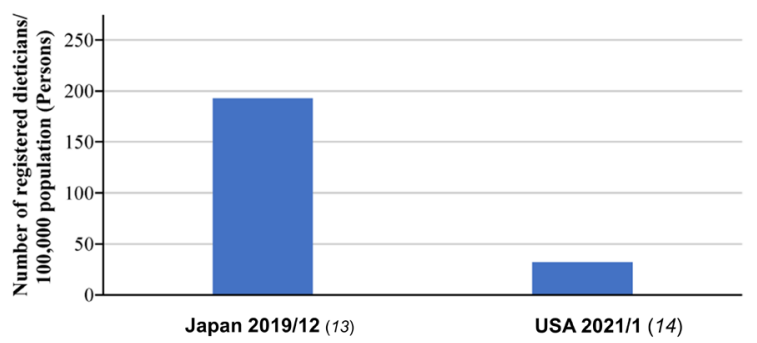

Figure 1. Comparison of the numbers of registered dieticians per 100,000 people in Japan and the USA. Compared to the USA, one of the most advanced countries in the world, the number of registered dietician per capita is significantly higher in Japan. Data source: Reference 13 for Japan, and Reference 14 for the USA.

early administration of enteral nutrition to ICU patients improves prognosis and significantly reduces the length of stay in the ICU and the length of hospitalization (9). Greater patient satisfaction is also seen. At least $85 \%$ of patients answered "satisfied" in a meal satisfaction survey conducted after registered dieticians collected and analyzed data from patients regarding meals, checking patients' dietary intake and taste preferences, and improving the menus (11).

"Nutrition management" is a system that is unique to Japan, where healthcare professionals' desire for patients to enjoy a safe, appetizing, and nutritious diet as part of Japan's excellent healthcare system is reflected. This can be considered an additional characteristic of Japan's healthcare system based on "patient-centeredness".

\section{Infection control}

The Ministry of Health, Labor, and Welfare defined healthcare-associated infections as: $i$ ) infections which patients are newly affected apart from the primary diseases in the healthcare facilities; $i$ ) infections which healthcare professionals, etc. have acquired in the healthcare facilities". We now present an outline of the measures against healthcare-associated infections concerning the first category.

Infection control refers to preventing the occurrence of infections and reducing the spread of infections when they do occur. It includes thoroughly implementing standard sterilization and launching countermeasures according to specific pathogens and clinical conditions as well as improving the hospital environment. These items are listed in the Centers for Disease Control and Prevention guidelines (15).

Infection controls were developed in Europe and the USA in response to an increase in antibiotic-resistant bacteria. Japan's countermeasures have been incorporated alongside those in Europe and the USA and have evolved into Japan's own system. Japan is working toward a system of infection control based on the concept of "patient-centeredness" in which countermeasures are made according to patients' respective conditions.

In Japanese hospitals, a special team comprising members from multiple disciplines is assembled to prevent infections and to implement anti-infection measures for the organization. Rather than uniformly following one standard guideline, each hospital customizes the guidelines to match its human resources, financial condition, and patient demographics. These guidelines incorporate efforts to improve work in clinical practice and are updated regularly. For example, a patient's shoes are removed and placed in a bag when they are put on a stretcher to prevent bacteria present on the soles of their shoes from coming into contact with the bed they lie on.

However, rather than making adherence to guidelines the primary goal, responses should be based on the guidelines while considering patients' needs. In some cases, hospitals even consider which zones should allow flowers to be brought in as get-well gifts for patients due to conditions such as immunosuppression. Even when such situations are not described in the guidelines, they are considered, according to the best healthcare service for patients in view of safety and patient satisfaction.

Thus, a flexible approach to infection control is taken in Japan with the best interests of the patients in mind. Consequently, accumulating the small efforts mentioned above has led to an appropriately hygienic environment unique to Japan. Many hospitals conduct surveillance regarding healthcare-associated infections in hospitals and have adopted a feedback system to improve their guidelines $(16,17)$. As a result of these infection controls in each hospital, the incidence of healthcare-associated infections (bloodstream infections/urinary tract infections/ventilation-related pneumonia) is low in Japan compared with European countries, which are considered to be advanced in this field $(17,18)$ (Figure 2).

In addition to the above-mentioned infection prevention measures, the proper use of antibiotic agents is promoted in Japan to reduce post-surgery deaths resulting from infections. In some cases, efforts are made to resolve delays in reporting test results, implementing appropriate treatments, and diminishing adverse events by cooperation among doctors, nurses, pharmacists, and clinical laboratory technicians and monitoring patients with culture-positive results for 24 hours a day, 365 days a year, based on the guidance published by the Ministry of Health, Labor and Welfare (10).

There is a reimbursement system for infection controls to promote these efforts. Since this system stipulates the measures against healthcare-associated infections to be taken as a requirement for calculating medical fees, each hospital across the country takes the appropriate measures against infection according to each situation. Some countries penalize medical institutions for not implementing infection control, while Japan evaluates the implementation of infection controls and provides additional remuneration as incentives that lead to quality improvement.

Consequently, Japan's system of "infection control" 


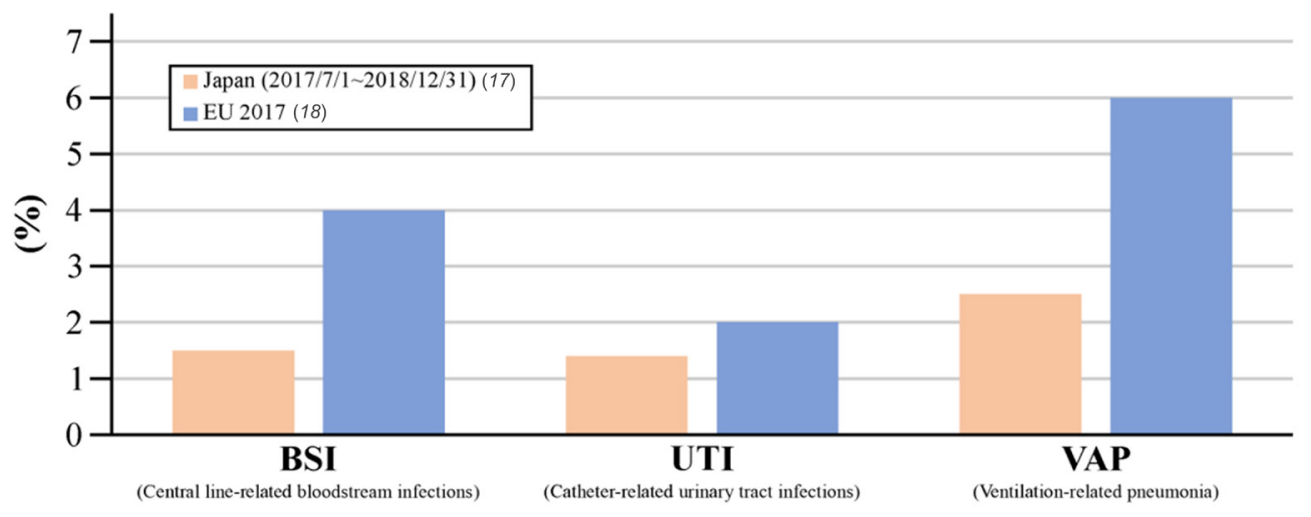

Figure 2. Comparison of the incidence of healthcare-associated infections according to clinical conditions in Japan and Europe. A comparison of the incidence of surgical site infections has been omitted due to a lack of comparable data. BSI, central line-related bloodstream infections; UTI, catheter-related urinary tract infections; VAP, ventilation-related pneumonia. Data source: Reference 17 for Japan, and Reference 18 for European countries.

Table 2. Comparison of 5-year survival rates for cancer treatments in the USA, Europe, and Japan

\begin{tabular}{lccccc}
\hline \multirow{2}{*}{ Categories } & \multicolumn{5}{c}{ Comparison of 5-year survival rates (\%) } \\
\cline { 2 - 6 } & Japan & USA & Germany & France & UK \\
\hline Lung cancer & 33 & 21 & 18 & 17 & 13 \\
Gastric cancer & 60 & 33 & 34 & 27 & 21 \\
Esophageal cancer & 36 & 20 & 21 & 14 & 16 \\
Colon cancer & 68 & 65 & 65 & 64 & 60 \\
Hepatic cancer & 30 & 17 & 13 & 18 & 13
\end{tabular}

"5-year survival rates for each type of carcinoma (including all stages) from 2010 to 2014. Data source: Reference 3.

is the most unique in the world as each hospital has repeatedly established their individual system based on systems adopted from Europe and the USA. This system could be regarded as a key characteristic of Japan's healthcare system based on the concept of "patientcenteredness".

Excellent treatment results from Japan's medical and healthcare system based on "patient-centeredness"

This report has discussed the nature of Japan's medical and healthcare system based on "patient-centeredness". Excellent treatment results for many diseases have also been observed in Japan based on the "correct diagnosis and optimal treatment". Here, we discuss the results of cancer treatments and minimally invasive medicine for cardiovascular diseases as specific examples.

\section{Cancer treatments}

Japan has strong outcomes in cancer treatment. Table 2 shows the five-year survival rates for various carcinomas in Japan, the USA, and some European countries (3). The five-year survival rates for treatment of major carcinomas are higher in Japan than those in the USA and the listed European countries. Many types of cancer treatments are available in Japan (Table 3).
Minimally invasive medicinal treatment for cardiovascular diseases

Japan also has excellent treatment results for minimally invasive medical treatment of cardiovascular diseases. Table 4 shows the 30-day mortality after treatment of ischemic heart diseases in Japan and in other countries as reference. The number of deaths due to cardiac arrest per 100,000 people in Japan, the USA, Germany, and France was $171,256,314$, and 182 , respectively (2). The number of deaths due to cardiac arrest per 100,000 people is lower in Japan than in other countries.

Minimally invasive medical treatments for a variety of cardiovascular diseases are also available in Japan (Table 5). Japan has better treatment results than Europe and the USA, particularly for cancer treatments and minimally invasive medical procedures for cardiovascular diseases. This is attributed to the excellent characteristics of Japan's medical and healthcare system, as well as the independently developed advanced healthcare system adopted from Europe and the USA based on "patient-centeredness" and ingenuity in clinical practice.

\section{Conclusion}

This report details examples of the systems within Japan's healthcare framework, namely "medical team approach," "nutrition management," and "infection controls," as well as treatment results using objective data. These three healthcare systems manifest the philosophy of "patientcenteredness," by which healthcare professionals always consider the ideal care and act accordingly. Another characteristic is that these healthcare systems are unique to Japan and were developed with the history, systems, and the culture of Japan.

Japan demonstrates positive treatment results comparable to Europe and the USA due to its rational medical system based on "patient-centeredness" and the pursuit of the "correct diagnosis and optimal treatment". 
Table 3. Cancer treatment methods and their results

\begin{tabular}{ll}
\hline Treatment methods & \multicolumn{1}{c}{ Number of cases (year) $^{*}$} \\
\hline $\begin{array}{l}\text { Laparoscopic surgery for cancers } \\
\text { (minimally invasive treatment for the digestive system) }\end{array}$ & $\begin{array}{l}\text { Stomach: } 15,000 \text { per year }(2018)(19) \\
\text { Lung: } 39,000 \text { per year }(2018)(19)\end{array}$ \\
Endoscopy/Endoscopic therapy & Stomach: 47,045 per year $(2017)(20)$ \\
$\quad$ ESD & Large intestine: $\geq 6,000(2017)(20)$ \\
$\quad$ EMR (including polypectomy) & Stomach: 3,824 per year $(2017)(20)$ \\
Hepatic cancer treatment (RFA) & 30,000 per year or more $(2014)(21)$ \\
IMRT & 25,000 per year $(2017)(22)$ \\
Particle-beam radiation therapy & 4,800 per year $(2017)(22)$
\end{tabular}

${ }^{*}$ The number of cases for each treatment method for Japan's population (126,020,000, as of 1/1/2020) is listed. EMR, endoscopic mucosal resection; ESD, endoscopic submucosal dissection; IMRT, intensity-modulated radiotherapy; RFA, radiofrequency ablation.

Table 4. The 30-day mortality after treatment for cardiac diseases in Japan compared with that in other countries

\begin{tabular}{lll}
\hline Treatment & \multicolumn{1}{c}{ Mortality in Japan (year) } & Mortality in other countries (year) \\
\hline Cardiac catheterization treatment & $0.05-0.2 \%(23)(2006)$ & USA: 1.20\% (24) (2005-2016) \\
Surgery for valvular heart diseases (TAVI) & $2.0 \%(25)($ October 2013-July 2015) & Germany: 2.40\% (26) (2012-2015) \\
Aortic stent grafting & & \\
$\quad$ Thoracic & Descending part: $1.40 \%(27)($ fiscal 2017) & USA \& Canada: 8.40\%* (28) (2013-2014) \\
& Arch: 3.0\% (28) (fiscal 2017) & USA \& Canada: $1.20 \%{ }^{* * *}(29)(2006-2015)$
\end{tabular}

"Analysis of 2,542 patients registered in the Vascular Quality Initiative compared with the mortality in Japan. ${ }^{* *}$ Analysis of 30,076 patients registered in the American College of Surgeons National Surgical Quality Improvement Program compared with the mortality in Japan.

Table 5. Treatment methods for cardiovascular diseases and their results

\begin{tabular}{ll}
\hline Treatment methods & \multicolumn{1}{c}{ Number of cases performed" } \\
\hline Cardiac catheterization treatment & Elective PCI: 192,670 per year (2018) (30) \\
Catheter ablation & 10,590 (August-December 2017) (31) \\
Surgery for valvular heart disease (TAVI) & 6,850 per year (2018) (30) \\
Aortic stent grafting & \\
$\quad$ Thoracic & 4,177 per year (2018) $(30)$ \\
Abdominal & 9,410 per year $(2018)(30)$ \\
"The number of cases for each treatment method for Japan's population $(126,020,000$, as of $1 / 1 / 2020)$. PCI, percutaneous coronary intervention; \\
TAVI, transcatheter aortic valve implantation.
\end{tabular}

This report intends to introduce Japan's medical and healthcare system based on "patient-centeredness" to healthcare providers worldwide for the global improvement of the quality of healthcare.

\section{Acknowledgments}

We would like to express our sincere gratitude to all participants who contributed to the study.

We received professional inputs from the following members including medical doctors in the management bracket in hospitals overseas: Dr. Minoru Akiyama (Medical Excellence JAPAN); Dr. Tianyuan Guo (Juntendo University Hospital); Dr. Masao Hashimoto (National Center for Global Health and Medicine); Dr. Dongcun Jin (Tsuyama Chuo Hospital International Medical Support Center); Dr. Takashi Karako (National Center for Global Health and Medicine); Dr. Dongmei
Ma (University of Tsukuba Hospital); Dr. Tetsuya Matsumoto (International University of Health and Welfare); Dr. Yasushi Miyazawa (Tokyo Medical University); Dr. Itaru Nakamura (Tokyo Medical University); Dr. Jun Oda (Tokyo Medical University); Dr. Haoying Shi (SinoUnited Health); Dr. Pham Tuan Anh (National Cancer Hospital of Viet Nam); and Dr. Zhaoqi Zhang (Meishen Medical Center).

Furthermore, we received valuable advice from the following members: Dr. Takao Aizawa (Japan Hospital Association), Dr. Yasuhiro Fujiwara (Pharmaceuticals and Medical Devices Agency), Dr. Kiyotaka Hoshinaga (Fujita Academy), Dr. Satoshi Imamura (Japan Medical Association), Dr. Yuko Kitagawa (Keio University), Dr. Norihiro Kokudo (National Center for Global Health and Medicine), Dr. Morito Monden (The Japanese Association of Medical Sciences), Dr. Yasuyuki Seto (The University of Tokyo), and Dr. Kotaro Yokote 
(National University Hospital Council of Japan).

\section{Funding: None.}

Conflict of Interest: The authors have no conflicts of interest to disclose.

\section{References}

1. Ministry of Health, Labour and Welfare. https://www. mhlw.go.jp/stf/seisakunitsuite/bunya/kenkou_iryou/ iryouhoken/iryouhoken01/index.html (accessed December 7, 2021). (in Japanese)

2. OECD. OECD health policy studies cardiovascular disease and diabetes policies for better health and quality of care. https://www.oecd.org/publications/cardiovasculardisease-and-diabetes-policies-for-better-health-andquality-of-care-9789264233010-en.htm (accessed March 6, 2021).

3. Allemani C, Matsuda T, Di Carlo V, et al. Global surveillance of trends in cancer survival 2000-14 (CONCORD-3): analysis of individual records for $37,513,025$ patients diagnosed with one of 18 cancers from 322 population-based registers in 71 countries. Lancet. 2018; 391:1023-1075.

4. Ministry of Health, Labour and Welfare. About promotion of medical team approach (Report of review conference regarding promotion of team approach). https://www. mhlw.go.jp/shingi/2010/03/dl/s0319-9a.pdf (accessed February 26, 2021). (in Japanese)

5. Coffey RJ, Richards JS, Remmert CS, LeRoy SS, Schoville RR, Baldwin PJ. An introduction to critical paths. Qual Manag Health Care. 2005; 14:46-55.

6. Japanese Society for Clinical Pathway. Overview. http:// www.jscp.gr.jp/about.html (accessed January 15, 2021). (in Japanese)

7. Hayakawa S. Organizational factors in the division of roles played by healthcare professionals in U.S. hospitals. http://www.ipss.go.jp/syoushika/bunken/data/ $p d f / 19455402 . p d f$ (accessed March 3, 2021). (in Japanese)

8. Shinoda M. Roles of doctors and nurses in France: focusing on the unique role of nurses. http://www.ipss. go.jp/syoushika/bunken/data/pdf/19455404.pdf (accessed March 7, 2021). (in Japanese)

9. Yanome H, Kawai C. Effects of intensive nutritional management in an intensive care unit on the length of stay and overall length of hospital stay: a retrospective analysis in a hospital. Nutrition Care and Management. 2019; 19:12-18. (in Japanese)

10. Medical Team Approach Promotion Council. Basic concept to promote medical team approach and collection of practical cases. https://www.mhlw.go.jp/stf/ shingi/2r9852000001ehf7-att/2r9852000001 ehgo.pdf (accessed December 10, 2020). (in Japanese)

11. Japan Council for Quality Healthcare. Hospital Function Evaluation Data Book 2018. https://www.jq-hyouka.jcqhc. or.jp/post/databook/4333 (accessed March 1, 2021). (in Japanese)

12. Daniela AN, Mona B, Ilan K, Avishay E, Karina C, Mursi G, Miriam L, Agnes G, Sigrid K, Mohamed M, Pierre S. Improved meal presentation increases food intake and decreases readmission rate in hospitalized patients. Clin Nutr. 2016; 35:1153-1158.
13. Ministry of Health, Labour and Welfare Health Bureau Health Division Nutrition Counselling Office. Changes in the number of registered dieticians. https://www. mhlw.go.jp/content/10901000/000358651.pdf (accessed December 7, 2021). (in Japanese)

14. Commission on Dietetic Registration. Registry statistics/ registered dietitian and registered dietitian nutritionist. https://www.cdrnet.org/registry-statistics (accessed January 26, 2021).

15. Centers for Disease Control and Prevention. Healthcareassociated Infections. https://www.cdc.gov/hai/index.html (accessed January 27, 2021).

16. Ministry of Health, Labour and Welfare. Japan nosocomial infections surveillance/Division of surgical site infection (SSI). https://janis.mhlw.go.jp/report/ssi.html (accessed December 20, 2020). (in Japanese)

17. Japanese Society for Infection Prevention and Control. Division of device-related infection surveillance. http://www.kankyokansen.org/modules/iinkai/index. php? content_id $=6$ (accessed December 28, 2020). (in Japanese)

18. European Centre for Disease Prevention and Control. Healthcare-associated infections in intensive care units - Annual Epidemiological Report for 2017. https:// www.ecdc.europa.eu/en/publications-data/healthcareassociated-infections-intensive-care-units-annualepidemiological-1 (accessed March 1, 2021).

19. Ministry of Health, Labour and Welfare. Statistics of medical care activities in public health insurance, 2018. https://www.mhlw.go.jp/toukei/saikin/hw/sinryo/tyosal8/ (accessed January 15, 2021). (in Japanese)

20. Ono H. The history, current status and future perspective of ESD for GI tract cancer. Nihon Shokakibyo Gakkai Zasshi. 2017; 114:971-977. (in Japanese)

21. The Japan Society of Hepatology. Clinical Practice Guidelines for Hepatocellular Carcinoma 2017. https:// www.jsh.or.jp/English/examination_en/guidelines_ hepatocellular_carcinoma_2017.html (accessed February 4, 2021).

22. JASTRO Japanese Society for Radiation Oncology. Database committee 2017 simplified structural survey. https://www.jastro.or.jp/medicalpersonnel/data_center/ cat6/cat1/post-6.html (accessed February 4, 2021). (in Japanese)

23. The Japanese Circulation Society. Guidelines for elective percutaneous coronary intervention in patients with stable coronary disease, JCS joint working group for guidelines for diagnosis and treatment of cardiovascular diseases (fiscal 2010 joint working group report) https://www.j-circ. or.jp (accessed July 26, 2021).

24. Bricker RS, Valle JA, Plomondon ME, Armstrong EJ, Waldo SW. Causes of mortality after percutaneous coronary intervention. Circ Cardiovasc Qual Outcomes. 2019; 12:e005355.

25. Shimura T, Yamamoto M. Results and future perspective of TAVI in Japan [Feature: Effectiveness and challenges]. https://www.jmedj.co.jp/journal/paper/detail.php?id=6515 (accessed July 26, 2021). (in Japanese)

26. Gaede L, Blumenstein J, Kim WK, Liebetrau C, Dörr O, Nef H, Hamm C, Elsässer A, Möllmann H. Trends in aortic valve replacement in Germany in 2015: transcatheter versus isolated surgical aortic valve repair. Clin Res Cardiol. 2017; 106: 411-419.

27. Japanese Committee for Stentgraft Management. Japanese Committee for Stentgraft Management/Therapeutic 
results. http://stentgraft.jp/pro/en/result/ (accessed March 5, 2021).

28. Scali ST, Feezor RJ, Neal D, Giles KA, Fatima J, Berceli SA, Huber TS, Beck AW. Predicting neurologic complications and 30-day mortality after thoracic endovascular aortic repair in the vascular quality initiative. J Vasc Surg. 2016; 63:559-560.

29. Yin K, Locham SS, Schermerhorn ML, Malas MB. Trends of 30-day mortality and morbidities in endovascular repair of intact abdominal aortic aneurysm during the last decade. J Vasc Surg. 2019; 69:64-73.

30. The Japanese Circulation Society. Report of the Japanese registry of all cardiac and vascular diseases 2018 Web version. https://j-circ.or.jp/jittai_chosa/jittai chosa2018web.pdf (accessed December 20, 2020). (in Japanese)
31. Japanese Heart Rhythm Society. J-AB registry, Japanese Heart Rhythm Society catheter ablation committee. http:// j-ab.ncvc.go.jp/(accessed March 6, 2021). (in Japanese)

Received August 24, 2021; Revised December 14, 2021; Accepted December 22, 2021.

Released online in J-STAGE as advance publication January 17, 2022.

*Address correspondence to:

Tatsuya Kondo, Medical Excellence JAPAN, Ichibancho Hougenzaka Bldg. 3F, 13 Ichibancho, Chiyoda-ku, Tokyo 1020082, Japan.

E-mail: yojigen@me-jp.org 